\title{
Mucopolysaccharidosis: Clinical and Radiological Aspects
}

\author{
Arshad Iqbal Wani, M.D., Khalid Farooqi, M.D., Mir Iftikhar Bashir, M.D., Shahnaz Mir, M.D., \\ Shariq R. Masoodi, D.M. \\ Department of Endocrinology, Sher-i-Kashmir Institute of Medical Sciences, Srinagar
}

\section{A B S T R A C T}

\begin{abstract}
Mucopolysaccharidosis (MPS) refers to a group of genetic disorder characterized by excessive accumulation of mucopolysaccharides secondary to deficiencies in specific enzymes. It produces characteristic skeletal abnormalities collectively termed as "dysostosis multiplex". Here we describe a young female child with classical radiological features of MPS on plain radiographs. JMS 2012;15(1):54-56.
\end{abstract}

Key Words: Mucopolysaccharidosis, skeletal abnormalities, dysostosis multiplex

A 27 months old female child was referred for evaluation of developmental delay, short stature and recurrent chest infections. The child was a product of non-consanguineous marriage and was born at term through normal vaginal delivery. The postnatal period was uneventful and the child appeared normal after birth. The parents got perturbed when they noticed poor growth, delayed achievements of milestones and delayed speech development.

On examination the patient had coarse facies(Figure 1). The weight was $10 \mathrm{~kg}$ and height was $80 \mathrm{~cm}$, both below the third percentile for her age. The circumference of the head was $48 \mathrm{~cm}$ (75th centile). Scalp and eyebrows were covered with abundant hair. The ears were low-set. Ophthalmologic examination revealed hypertelorism and a fine corneal clouding in both eyes. The nose was broad with a flat base, and macroglossia was present. The neck was short and supple. Examination of oral cavity revealed a high arched palate and macroglossia. The abdomen was protuberant and

\section{Correspondence:}

Dr. Shariq R Masoodi

Additional Professor

Department of Endocrinology

Sher-i-Kashmir Institute of Medical Sciences, Srinagar

E-Mail:Shariq.masoodi@gmail.com

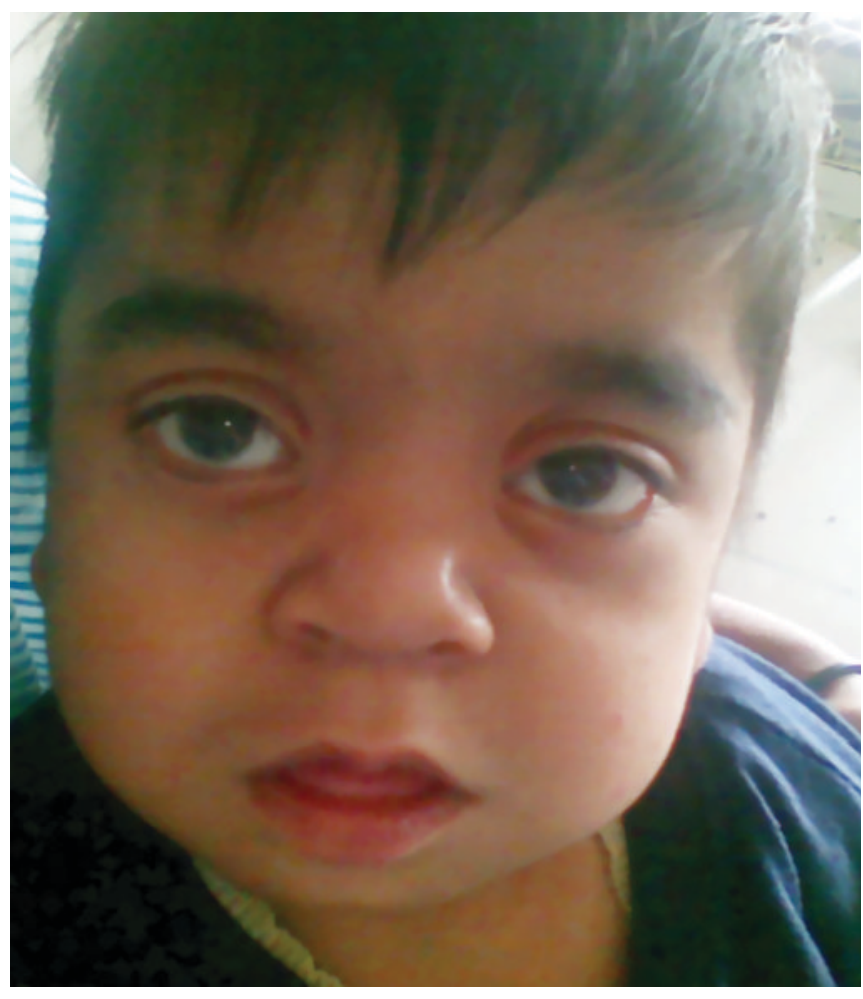

FIGURE 1: Depressed nasal bridge, hypertelorism, corneal clouding 
liver was palpable $4 \mathrm{~cm}$ below the right costal margin; spleen was palpable $3 \mathrm{~cm}$ below left costal margin. The anteroposterior diameter of the chest was increased with a prominent sternum and the lungs were clear on auscultation. Flexion deformity was present at elbow and knee joints. Cardiovascular examination was unremarkable and Echocardiography study was normal

Skeletal survey revealed features suggestive of skeletal dysostosis (Figures 2- 7) and a clinical impression of MPS (Hurler syndrome) was made however urinary glycosaminoglycans level could not be obtained.

\section{Discussion}

The mucopolysaccharidoses (MPS) are a heterogeneous group of lysosomal storage disorders caused by the deficiency of enzymes required for the stepwise breakdown of glycosaminoglycans. ${ }^{1}$ Hurler syndrome is one of the commonest type of mucopolysaccharidoses and is inherited in an autosomal recessive manner. The incidence of hurler syndrome is approximately one in 100,000 births while the estimated total incidence of all types of MPS is approximately one in 20,000 live births. ${ }^{2}$ Early enzyme replacement therapy is critical to avoid the development of significant morbidity.

Hurler syndrome is characterized by normal appearance at birth with gradual development of coarse facies and midface hypoplasia. Other features include: hernias, recurrent chest and sinus infections, hepatosplenomegaly, hydrocephalus, unrecognized sleep apnea, cardiac abnormalities like cardiomyopathy, valvular regurgitation and short stature (short trunk). ${ }^{3}$ The characteristic feature of the disorder is corneal clouding which is caused by structural changes in the corneal stroma and is an important cause for blindness. The disease is also characterized by presence of typical skeletal complex-typical dysostosis multiplex. The average age at death is 5 years.

The long bones are short and thick and show swelling of their central aspects due to widening of their medullary canal. The iliac bones are flared and show coxa valga and flattened acetabulum. The metacarpals narrow proximally and widen distally with irregular ends giving rise to "bullet deformity". Hypoplasia of the anterosuperior areas of the

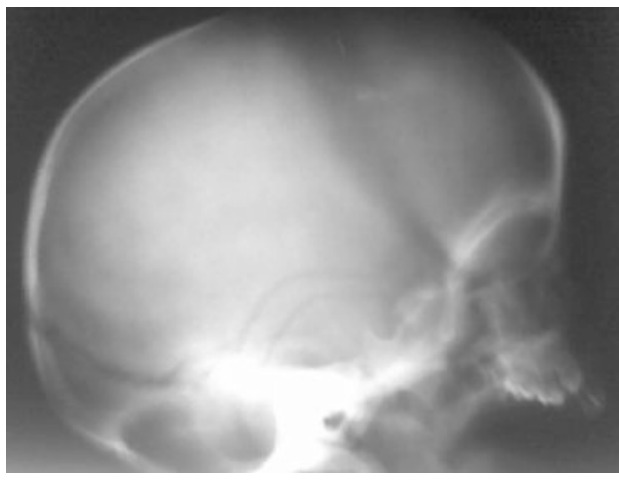

FIGURE 2: Lateral X ray of skull showing J shaped sella turcica

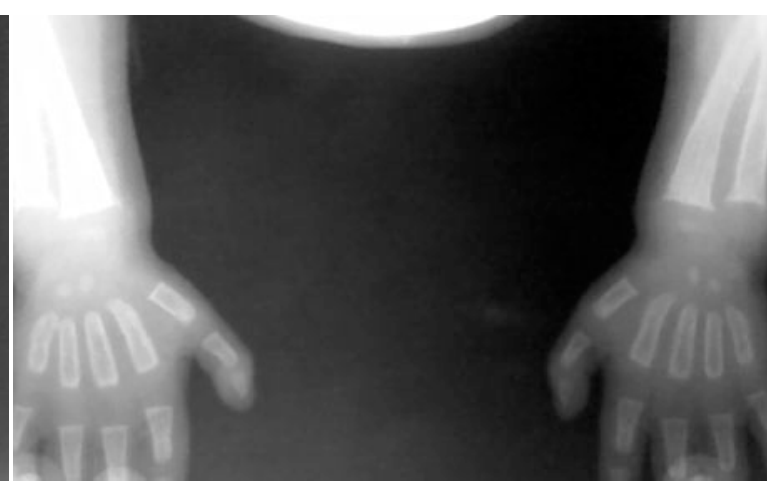

FIGURE 3: $\mathrm{X}$ ray of hand showing bullet shaped metacarpals and phalanges with proximal narrowing

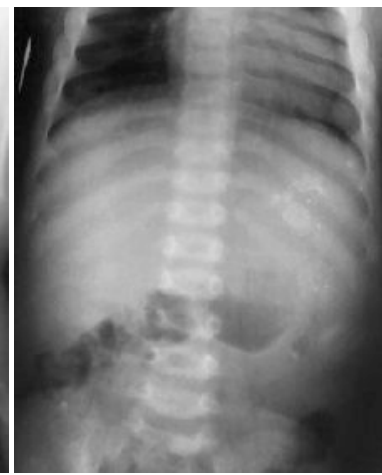

FIGURE 4: AP view of dorsolumbar spine showing platyspondyly

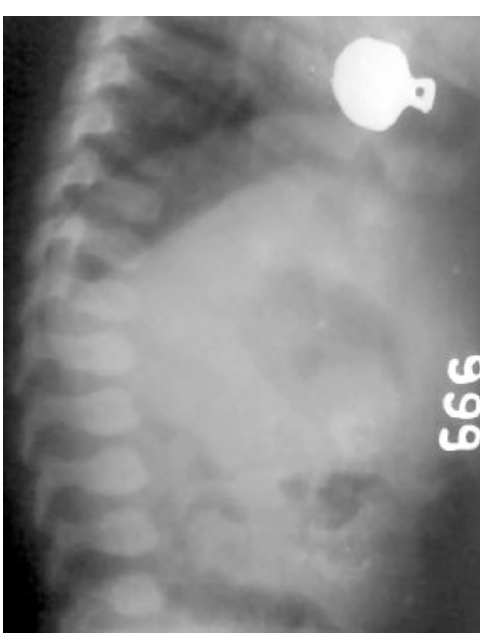

FIGURE 5: Lateral $x$ ray of dorsolumbar spine showing anterosuperior hypoplasia of the lumber vertebral bodies with prominent inferior beaks

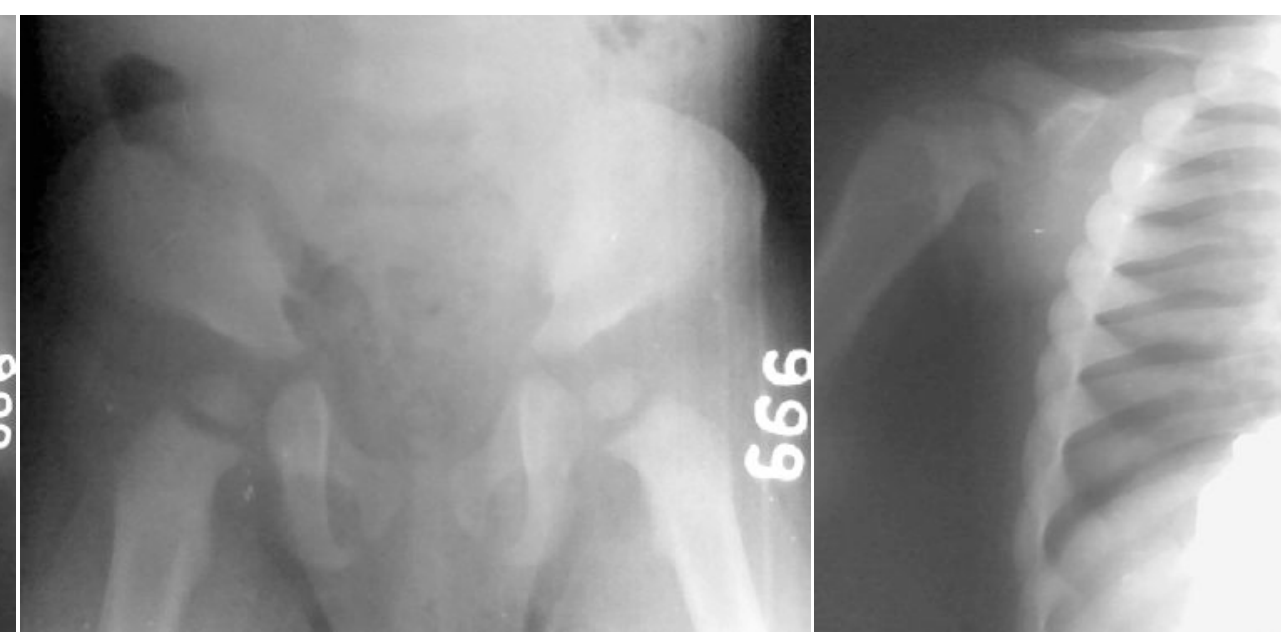

FIGURE 6: $X$ ray of pelvis AP view showing hypoplastic pelvis and poorly formed femoral head
FIGURE 7: $X$ ray of chest PA view showing narrowing of ribs at the vertebral ends and widening at sternal ends (oar-shaped). 
lower thoracic and upper lumbar vertebral bodies results in characteristic beaking appearance deformity. The ribs have a characteristic "oar shape" due to narrowing at the vertebral column and broadening at the sternal end. The skull is large and deformed due to craniosynostosis. Sella is typically "j" or boot-shaped and results from extension of the anterior recess underneath the anterior clinoid process. ${ }^{4}$

Radiological investigations play a very important part in the diagnostic workup of such patients as can be seen from this case description and the accompanying figures.

\section{References}

1. The mucopolysaccharidoses Whitley C. In: McKusick's Heritable Defects of Connective Tissue, Beighton, P (Ed) Mosby, St. Louis, 1993. p.367.
2. Malm G, Lund AM, Mansson JE, Heiberg A. Mucopolysaccharidoses in the Scandinavian countries: incidence and prevalence. Acta Paediatr 2008;97(11): 1577-81.

3. Dangel JH. Cardiovascular changes in children with mucopolysaccharide storage diseases and related disorders-clinical and echocardiographic findings in 64 patients. EurJ Pediatr 1998;157(7):534-8.

4. Wappner RS. Lysosomal storage disorders. In: Oski's Pediatrics. Principles and Practice, 4th ed, McMillan, JA, Feigin, RD, DeAngelis, C, Jones, MD (Eds), Lippincott, Williams Wilkins, Philadelphia 2006. p. 2199. 